\title{
Species-independent analytical tools for next-generation agriculture
}

\author{
Tedrick Thomas Salim Lew ${ }^{1}$, Rajani Sarojam ${ }^{2,3}$, In-Cheol Jang $\oplus^{2,3}$, Bong Soo Park $\oplus^{2,3}$, \\ Naweed I. Naqvi' ${ }^{2}$ Min Hao Wong', Gajendra P. Singh'3, Rajeev J. Ram ${ }^{3,4}$, Oded Shoseyov ${ }^{5}$, \\ Kazuki Saito ${ }^{6}$, Nam-Hai Chua ${ }^{2,3 凶}$ and Michael S. Strano $\oplus^{1,3 凶}$
}

\begin{abstract}
Innovative approaches are urgently required to alleviate the growing pressure on agriculture to meet the rising demand for food. A key challenge for plant biology is to bridge the notable knowledge gap between our detailed understanding of model plants grown under laboratory conditions and the agriculturally important crops cultivated in fields or production facilities. This Perspective highlights the recent development of new analytical tools that are rapid and non-destructive and provide tissue-, cell- or organelle-specific information on living plants in real time, with the potential to extend across multiple species in field applications. We evaluate the utility of engineered plant nanosensors and portable Raman spectroscopy to detect biotic and abiotic stresses, monitor plant hormonal signalling as well as characterize the soil, phytobiome and crop health in a non- or minimally invasive manner. We propose leveraging these tools to bridge the aforementioned fundamental gap with new synthesis and integration of expertise from plant biology, engineering and data science. Lastly, we assess the economic potential and discuss implementation strategies that will ensure the acceptance and successful integration of these modern tools in future farming practices in traditional as well as urban agriculture.
\end{abstract}

G lobal demand for agricultural products is anticipated to increase rapidly in the next few decades, fuelled by a growing world population and greater per capita income $e^{1-3}$. However, biotic and abiotic environmental stresses, such as plant pathogens, sudden fluctuations in temperature, drought, soil salinity and toxic metal pollution, impair crop productivity and lead to significant losses in agricultural yield worldwide $\mathrm{i}^{4,5}$. Crop pathogens and insect pests alone cause an estimated global yield loss of $11-30 \%$ for five major crops of global importance-wheat, rice, maize, potato and soybean-with the highest crop losses observed in regions already suffering from food insecurity ${ }^{6,7}$. The effects of climate change, which increase the frequency of extreme weather and plant disease outbreaks, impose additional pressure on agricultural innovation to meet the growing demand ${ }^{8-11}$. Against this backdrop, there has been an increasing demand for sustainable agricultural practices to produce crops with higher yield and quality ${ }^{12-14}$. Farming in controlled environments, such as urban containers or rooftop farms, has demonstrated that the throughput of plant biomass can be significantly increased via the control of microclimates ${ }^{15,16}$. It has been noted that the outbreak of the COVID-19 pandemic in 2020 serves as a humble reminder of the pivotal role that urban farms can play in mitigating food supply disruptions within a city ${ }^{17}$. The growing importance of agriculture to meet the global food demand requires a concomitant increase in our understanding of how plants grow and adapt outside of the laboratory for these farming practices to be effective.

During the past few decades, significant progress has been made in understanding plant growth, signalling pathways and their adaptive responses to environmental stresses in the model plant Arabidopsis thaliana ${ }^{18-20}$. Detailed genomic data and quantitative trait loci (QTL) can be found in global repositories such as The Arabidopsis Information Resource (TAIR) ${ }^{21}$, providing a wealth of resources that enable versatile manipulation of $A$. thaliana genes to study plant development and physiology $\mathrm{y}^{22,23}$. Transgenic plants with genetically encoded biosensors allow for in vivo study of the dynamics of plant signalling molecules, which can be monitored with imaging platforms in a laboratory setting ${ }^{24}$. While these advances have been instrumental in advancing our understanding of plant science, our knowledge of plant behaviour in the field remains more limited. This is primarily due to the difficulty in extending genetic engineering approaches to study agriculturally important plants, and the dearth of alternative tools to probe the internal plant state $^{25,26}$. Current genomic data in A. thaliana or unicellular green algae such as Chlamydomonas often cannot be used to predict or represent the complexities in agriculturally relevant $\mathrm{crops}^{27}$. There are genomic resources available for model crops such as rice (Oryza sativa), maize (Zea mays) and tomato (Solanum lycopersicum) $)^{28-30}$, but these denote only a handful of families and do not sufficiently represent the biodiversity of crops $^{31}$. In addition, studying the signalling pathways and physiology of these crops under variable field conditions remains a longstanding challenge ${ }^{32}$. Measuring the level and temporal dynamics of metabolites in these crops typically relies on mass spectrometry-based techniques ${ }^{33-35}$. These methods are destructive and require laborious processing of multiple samples of plants per data point. Transcriptomic data of crops under field conditions remain scarce due to the complexity of such studies ${ }^{36}$. These challenges create a gap between our detailed understanding of model plants and important agricultural targets for humans, contributing to our fragmentary knowledge of the underpinning

'Department of Chemical Engineering, Massachusetts Institute of Technology, Cambridge, MA, USA. ${ }^{2}$ Temasek Life Sciences Laboratory, 1 Research Link, National University of Singapore, Singapore, Singapore. ${ }^{3}$ Disruptive \& Sustainable Technologies for Agricultural Precision, Singapore-MIT Alliance for Research and Technology, Singapore, Singapore. ${ }^{4}$ Research Laboratory of Electronics, Massachusetts Institute of Technology, Cambridge, MA, USA. ${ }^{5}$ The Faculty of Agriculture, Food and Environment, The Hebrew University of Jerusalem, Rehovot, Israel. ${ }^{6}$ Metabolomics Research Group, RIKEN Center for Sustainable Resource Science, Yokohama, Japan.凶e-mail: chua@mail.rockefeller.edu; strano@mit.edu 
physiological processes that enable crops to adapt and grow in agricultural conditions.

In recent years, there has been significant progress towards new innovative tools which can be applied to probe the spatiotemporal profile of plant chemical signals in model and non-model plants alike, providing a potential mechanistic bridge between the two. Such species-independent platforms can also be applied non-destructively and thus enable real-time investigation of plant physiology and signalling. This Perspective reviews the underlying elements of such next-generation tools and discusses how they can bridge the gap between model plants in the laboratory and field application for agriculturally relevant plants. We also provide a future outlook for the integration of these tools with existing technologies to transform crop improvement and highlight what needs to be done to increase the utility and accessibility of these tools to the end-users.

\section{Limitations in current plant phenotyping tools}

Recent advances in imaging systems have led to the development of field-based phenotyping platforms to collect high-throughput trait measurements over a large field of view ${ }^{37-39}$. With these systems, plants' physical and physiological features can be interrogated non-destructively ${ }^{40}$. Unmanned aerial vehicles (UAVs) now allow plant phenotyping and disease monitoring at the spatial resolution of an individual plant ${ }^{41,42}$. They can be paired with portable analytical devices and smartphone-based platforms to provide remote sensing data ${ }^{43,44}$. These technologies are currently being used to extract information about crop health and environmental stresses based on plant trait measurement in terms of chlorophyll concentration, water potential, leaf area and temperature ${ }^{45-48}$. While field-based phenotyping platforms convey useful information about crop health status and can be applied over a large area, their effective implementation often requires clear phenotypic manifestation that has already altered the plants' spectral profile. The time lag between stress perception and phenotypic changes required for detection may render timely intervention difficult and reduce the effectiveness of management decisions for certain or specific types of stress conditions. For instance, the measurement of hyperspectral images using drones may only show a warning when plants are already dehydrated or infected ${ }^{49}$. Yang et al. evaluated several examples of high-throughput phenotypic screening applied in A. thaliana or crops $^{50}$. These methods include ground-based phenotyping in the field (for example, crop phenology recording system (CPRS) ${ }^{51}$ and field scanalyzer ${ }^{52}$ ), remote sensing via drones, post-harvest phenotyping (for example, seed-evaluation accelerator (SEA ${ }^{53}$ and phenoSeeder ${ }^{54}$ ) and pocket phenotyping. Most of these methods were determined to be too costly at present, of low measuring speed in their current form or of limited resolution. In addition, they still rely on phenotypic changes such as a decrease in chlorophyll concentration and plant water potential, both of which can be caused by manifold stress types ${ }^{55}$. This type of detection method therefore lacks specificity, and early intervention to remediate the specific stresses remains challenging.

Another potential issue with the deployment of remote sensor technologies in a field setting is the question of how many sensor data points is enough to obtain a statistically representative view of the field. Varella et al. described the use of data acquisition within the precision farming context in a regular grid as well as directed grid format, depending on the end goal and applications ${ }^{56}$. Images collected by remote sensors make it possible to obtain a great deal of information about specific 'targets' on the surface, including qualitative and quantitative aspects, such as shape, geographical context, temporal behaviour, size, physical properties, intrinsic aspects and/ or state-specific factors. However, data management is still challenging, and calibration is almost always required to adjust for changes in canopy structure, camera geometries, incident sunlight angle and other uncontrollable environmental factors ${ }^{43}$. The large image data also necessitates complex data interpretation, limiting the automation of image processing ${ }^{57}$. Most commercial image analysis software and its built-in algorithms are not open source and cannot be modified easily across different platforms ${ }^{50}$. As such, they are often not accessible in their current form to smaller farms $\mathrm{s}^{58}$, and the underlying concern of whether these techniques are capable of early diagnosis of stresses or nutrient deficiency persists.

\section{Species-independent tools for early detection of plant stresses}

Plants transmit various biotic and abiotic stress signals to reprogram the cellular transcriptional machinery and metabolic responses to enhance their fitness under stress ${ }^{59}$. Here, we briefly review two different technologies for monitoring these stress signals: nanosensors for measurements within the plant organs and electrical sensors for measuring surface biopotentials. Both of these sensors monitor stress signals at short time-scales-from seconds to minutes. At intermediate time-scales, the stress response manifests as changes in metabolites. Here, we consider spectroscopic monitoring of metabolites within the plant and volatile organics that the plants produce within hours as part of their stress response. While some biotic stresses such as vector-borne viruses and vascular pathogens require only a short window for infection with very limited management options ${ }^{60,61}$, the information obtained from nanoscale and spectroscopic measurements may be used to aid remediation strategies to mitigate certain stresses such as water and nutrient availability, as well as moderate changes in the abiotic environment.

Perception of stress stimuli involves the propagation of short-lived signalling molecules, such as reactive oxygen species (ROS) and intracellular calcium $\left(\mathrm{Ca}^{2+}\right)$, and phytohormones, such as jasmonates, salicylates, auxins, gibberellins and abscisic acid ${ }^{62-64}$. Signal transduction triggers a cascade of transcriptional reprogramming that changes cellular development, plant growth and behaviour $^{65,66}$. Key signalling molecules and phytohormones are conserved across species, albeit with potentially different spatiotemporal dynamics ${ }^{67-70}$. Monitoring the evolution of plant signalling molecules as they are generated upon stress perception has the potential to enable early diagnostics of environmental or biological stressors. If these indicators can be intercepted before the manifestation of stress-induced phenotypic changes, there is potential for intervention to prevent the yield loss and reduction in crop quality. This will necessarily depend on the type and magnitude of stress as well as the availability of management options. While detecting a static concentration of a signalling molecule may give an indication of plant health, such information is not as useful as the concentration profile of the signalling molecule in space and time. Hence, to develop an effective tool capable of studying plant physiology and reporting stress events, both the sensitivity and the temporal resolution of the platform have to be considered. In addition, these tools have to be stable in terms of their structural integrity and performance, and they should give a clear output that can be easily interpreted with portable electronics or devices commonly used by agricultural end-users.

There have been some promising developments in the engineering and application of new analytical tools to study plants in agriculturally relevant settings. Nanomaterials, defined as inorganic or organic matter with dimensions smaller than $100 \mathrm{~nm}$, have unique physical and optical properties that can be harnessed for in vivo detection of plant signalling molecules ${ }^{71,72}$. Some nanoparticles, such as single-walled carbon nanotubes (SWNT), have photostable emission in the near-infrared region (NIR) away from the chlorophyll autoflorescence ${ }^{73}$. The sensitivity and selectivity of nanomaterials can also be engineered via facile modification of their surface chemistry ${ }^{71,74}$. These nanosensors can provide access to real-time information about plant health through non-destructive 
a
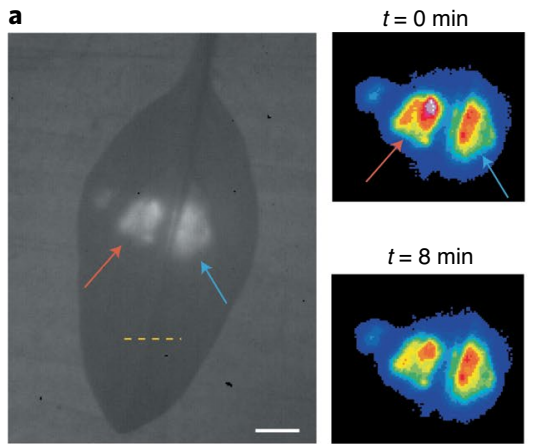

$t=8 \min$

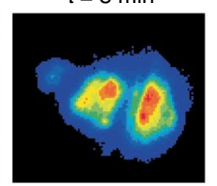

$t=1 \min$

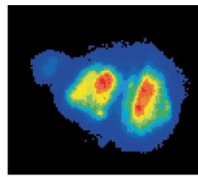

$t=10 \mathrm{~min}$

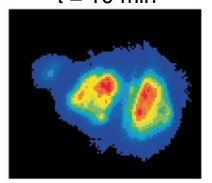

$t=2 \min$

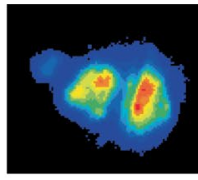

$t=15 \mathrm{~min}$

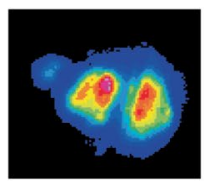

$t=4 \min$

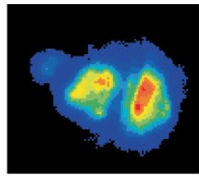

$t=20 \mathrm{~min}$

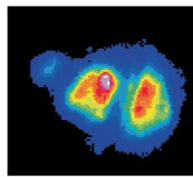

$\mathrm{nIR}$

intensity

b

c
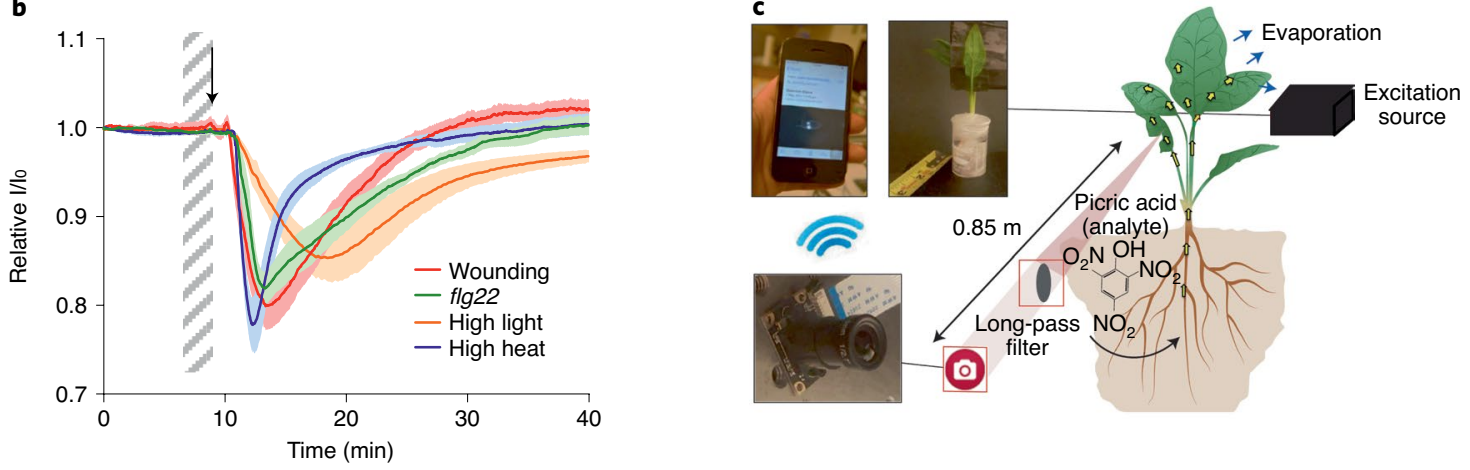

d

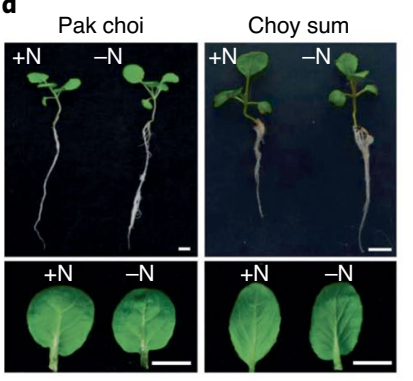

e

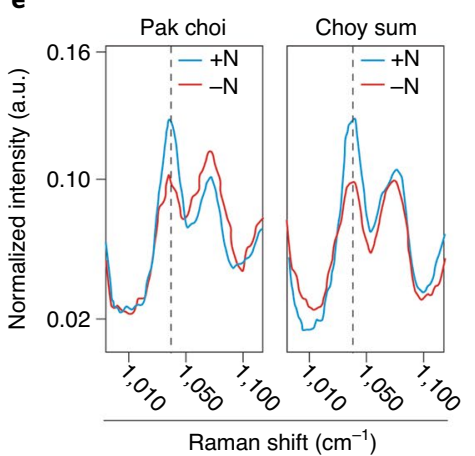

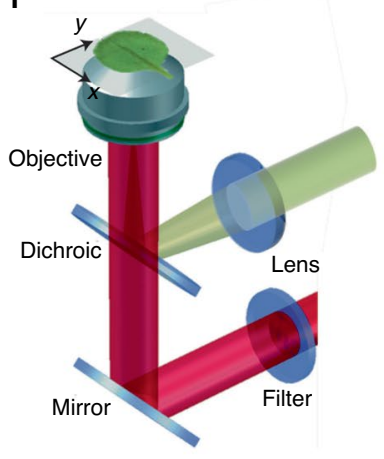

Fig. 1 | First-generation examples of optical nanosensors and Raman technology for non-destructive monitoring of plant stresses. a, Bright-field image of plant infiltrated with SWNT nanosensors (left panel). Red and blue arrows indicate active and reference nanosensors, respectively; the dashed line represents the wounding position. Scale bar, $5 \mathrm{~mm}$. False-coloured images show the post-wounding sensor dynamics tracking the transient $\mathrm{H}_{2} \mathrm{O}_{2}$ wave (right panel). b, Nanosensors' response to wounding, flg22, high-light and heat-stress treatments. The black arrow represents the time point at which stress is applied ( $t=10 \mathrm{~min}$ ), except high-light stress, which was applied for $2 \mathrm{~min}$ (dashed region). Panels $\mathbf{a}$ and $\mathbf{b}$ reproduced from ref. ${ }^{76}$, Springer Nature Ltd. c, Schematic of the experimental setup for the standoff detection of nitroaromatics in plants using nanobionics. The information from the nanosensors embedded on the plant leaf is relayed to a portable Raspberry Pi-based electronic device. Adapted from ref. ${ }^{77}$, Springer Nature Ltd. $\mathbf{d}$, Morphological phenotype of two-week-old seedlings of pak choi (Brassica rapa chinensis) and choy sum (Brassica rapa var. parachinensis) transferred into nitrogen-sufficient $(+\mathrm{N})$ or nitrogen-deficient $(-\mathrm{N})$ hydroponic medium, grown for $3 \mathrm{~d}$ and studied by Raman spectroscopy. Scale bars, $1 \mathrm{~cm}$. e, Raman spectral analysis of early nitrogen deficiency in leafy vegetables, pak choi and choy sum. f, Fibre-optic-enabled Raman spectroscopy system used for field portable applications. Panels $\mathbf{d}-\mathbf{f}$ reproduced from ref. ${ }^{94}$ under a Creative Commons licence (http://creativecommons.org/licenses/by/4.0/).

monitoring of endogenous signalling molecules and plant hormones. They can also be introduced to a wide range of plant species currently not amenable to genetic modification ${ }^{75}$. We recently demonstrated the application of SWNT to study stress-induced hydrogen peroxide $\left(\mathrm{H}_{2} \mathrm{O}_{2}\right)$ signalling waves in seven different plant species $^{76}$. Nanosensors were embedded into leaf mesophyll cells via needleless syringe infiltration. Upon contact with $\mathrm{H}_{2} \mathrm{O}_{2}$, the nanosensors' NIR fluorescence intensity was quenched reversibly, and the intensity modulation could be monitored at a remote distance in real time (Fig. 1a). The wave characteristics of the $\mathrm{H}_{2} \mathrm{O}_{2}$ signalling pathway probed by the nanosensors were found to be different across species and specific for the stresses that plants perceived (Fig. 1b). Such demonstration showcases the utility of speciesagnostic tools to help elucidate the complex plant signalling pathways during growth and acclimation to external conditions in both model and non-model plants. In another study, peptide-functionalized SWNT were engineered to serve as an optical reporter of nitroaromatic concentrations in a spinach (Spinacia oleracea) plant (Fig. 1c) $)^{77}$. Quantum dots are another class of versatile nanomaterials with tuneable emission intensity, ranging from the visible to 
the $\mathrm{NIR}^{78}$. Functionalized quantum dots have been shown to detect exogenously introduced glucose in A. thaliana and algae C. zeylan$\mathrm{ica}^{79}$. The information obtained by SWNT- and quantum dot-based nanosensors can further be conveyed using a portable Raspberry $\mathrm{Pi}$-based camera and platform at remote distances. Fluorescent carbon dots have emerged as a promising substitute to conventional quantum dots due to their excellent biocompatibility ${ }^{80}$. Carbon dots have been used to detect heavy metal pollution in the cell walls of onion $^{81}$. While these studies were conducted in laboratory settings, they highlight proven possibilities for next-generation technologies to empower future agricultural practices.

An emerging class of sensor technologies that can be conveniently applied to monitor plant growth and dynamics is wearable sensors. The measurement of bioelectric potential changes in plants can be used to detect changes in environmental factors such as atmospheric pressure, temperature, humidity and light intensity $^{82}$. Ochiai et al. compared commercially available boron-doped diamond (BDD), silver and platinum plate electrodes for measuring electrochemical signals in potted Opuntia hybrid plants and also in three different ground-planted trees ${ }^{83}$. The BDD electrodes were found to be 4-7 times more sensitive to bioelectric potential changes in potted Opuntia hybrid plants compared to platinum or silver electrodes. Similarly, for ground-planted trees, where bioelectric potential change was induced by changing temperature and humidity for months without replacing, removing or changing electrodes, BDD electrodes were 5-10 times more sensitive than platinum electrodes. Thus, the BDD electrode-based monitoring system has the potential to measure live plant growth and may represent a promising warning system for suddenly changing environmental conditions.

The stress signals that have been discussed previously in this section trigger metabolic responses in the plant. Metabolomics is becoming increasingly common in plant physiology and biochemistry, and to date has been applied to studies dealing with plant stresses including water, temperature, light, nutrient, oxidative and biotic stresses ${ }^{84-87}$. To identify, quantify and characterize metabolites requires high-throughput analytical technologies. Several techniques including gas chromatography-mass spectrometry (GC-MS), liquid chromatography (LC)-MS, capillary electrophoresis (CE)-MS and nuclear magnetic resonance spectroscopy (NMR) are commonly used in plant metabolomics research ${ }^{88-91}$. Unfortunately, these widely used techniques require sample preparation and are not suitable for in vivo monitoring of metabolites. If our goal is to monitor plant stress to optimize crop yield, techniques that require destruction of the plant and lengthy sample preparation fail to realize the potential of metabolomics to provide real-time phenotypic signatures.

Raman spectroscopy enables non-destructive biochemical detection capable of interrogating multiple molecular species simultaneously. As an optical tool, Raman spectroscopy can be considered as an extension of the imaging techniques that are already widely deployed in field-based phenotyping. However, Raman spectroscopy allows for significantly greater chemical specificity. To date, several groups have applied Raman spectroscopy for monitoring biotic and abiotic stresses. Early reports utilize Raman spectroscopy for measurements of metabolites such as carotenoids and anthocyanins as markers for abiotic stress in plants-including drought, saline, light and heat stress ${ }^{92}$. Similarly, the carotenoid peaks were observed as early stress markers for viral infections ${ }^{93}$. While these studies were performed on a few specimens of an ornamental plant (coleus lime or Abutilon hybridum), we can imagine that a high-throughput tool for monitoring plant stress in real time could benefit both plant biologists and farmers. Indeed, we have recently observed the nitrate peak in vegetable crops using NIR Raman spectroscopy ${ }^{94}$. Early diagnosis and specific diagnosis of nitrogen deficiency was observed in A. thaliana as well pak choi (Brassica rapa chinensis) and choy sum (Brassica rapa var. parachinensis). In all cases, the nitrate Raman line was observed to decrease well before any changes in leaf reflectance or chlorophyll concentration (Fig. 1d-f). These changes were observed early enough for nitrogen addition to recover the full yield of the leafy vegetables. Similarly, early detection of infection in important crop species by Raman spectroscopy has progressed rapidly in the last few years ${ }^{95}$. Since Raman is probing the optical properties of the plant leaf, it will likely be feasible to perform these measurements remotely from distances as great as $100 \mathrm{~m}$ (refs. ${ }^{96,97}$ ). As the cost of Raman hardware declines, metabolite measurements could become an everyday part of agriculture and the food supply-from farm to market to table.

Plants also emit characteristic volatile organic compounds (VOCs), which play a pivotal role in plant growth, communication and defence ${ }^{98,99}$. VOC profiling can give an indication of the health status of high-value commodity crops and provide new insights into interplant and plant-to-insect communication ${ }^{100}$. Polymeric sorbent traps are usually employed to concentrate VOCs before they are sent to the laboratory for thermal desorption and analysis with MS-based systems ${ }^{101}$. A trace amount of VOCs can be detected with this platform, but it requires bulky and expensive instrumentation for direct in-field analysis ${ }^{100}$. Zheng et al. recently introduced the integration of plasmonic nanoparticles in a smartphone-based VOC fingerprinting platform for the detection of Phytophthora infestans in tomato plants ${ }^{102}$. The sensor array is composed of gold nanoparticles with different aspect ratios which aggregate upon VOC contact, triggering a change in their colorimetric profile. When tested in a greenhouse setting, the smartphone-based sensor array was able to capture the pathogen-induced VOC changes in tomato leaves as early as two days post-infection before visible symptoms appeared. In another demonstration, Fong et al. reported a SWNT-based chemiresistive sensor capable of detecting $15 \mathrm{ppb}$ concentration of ethylene within seconds of exposure ${ }^{103}$. Ethylene is an important VOC that is central to the regulation of plant development, ranging from seed germination to fruit ripening and senescence. The sensor was able to monitor dynamic changes in trace amounts of ethylene during the senescence of carnation and lisianthus flowers. Artificial electronic nose (E-nose) platforms are also being developed for rapid, non-invasive monitoring of plant $\mathrm{VOCs}^{104}$. They comprise of a sensor array, signal conditioning circuit and pattern recognition algorithms. However, issues related to selectivity and the effect of environmental conditions necessitates further improvement to such sensor systems for optimal detection. VOC sensors do not need to be implanted within the plant tissues, but they have to show high sensitivity to capture trace amounts of VOCs commonly emitted by plants. In addition, their long-term stability, reproducibility, structural integrity and robustness will have to be considered for in-field analysis.

\section{New opportunities afforded by species-independent tools}

We propose that the newly developed tools introduced above can facilitate a new understanding of crop behaviour and dynamics in agricultural settings. The adoption of the aforementioned technologies in the study of model plants and their genetic variants can help validate the utility of these tools. In addition, they can also complement research in plant biology, especially in instances where genetically encoded biosensors are limited in utility; for example, in multiplexing and imaging of various molecules simultaneously. CRISPR-Cas9 systems, especially those that can produce transgene-free genome-edited crops and thus circumvent regulatory concerns in many countries ${ }^{105,106}$, could also be used in concert with advanced analytical technologies to aid trait identification and alteration for improved crop performance. Enhancing our basic understanding of plant science can bridge the gap between model and non-model plants, and ultimately help to extend the applicability of these tools for plants outside of laboratory settings. 

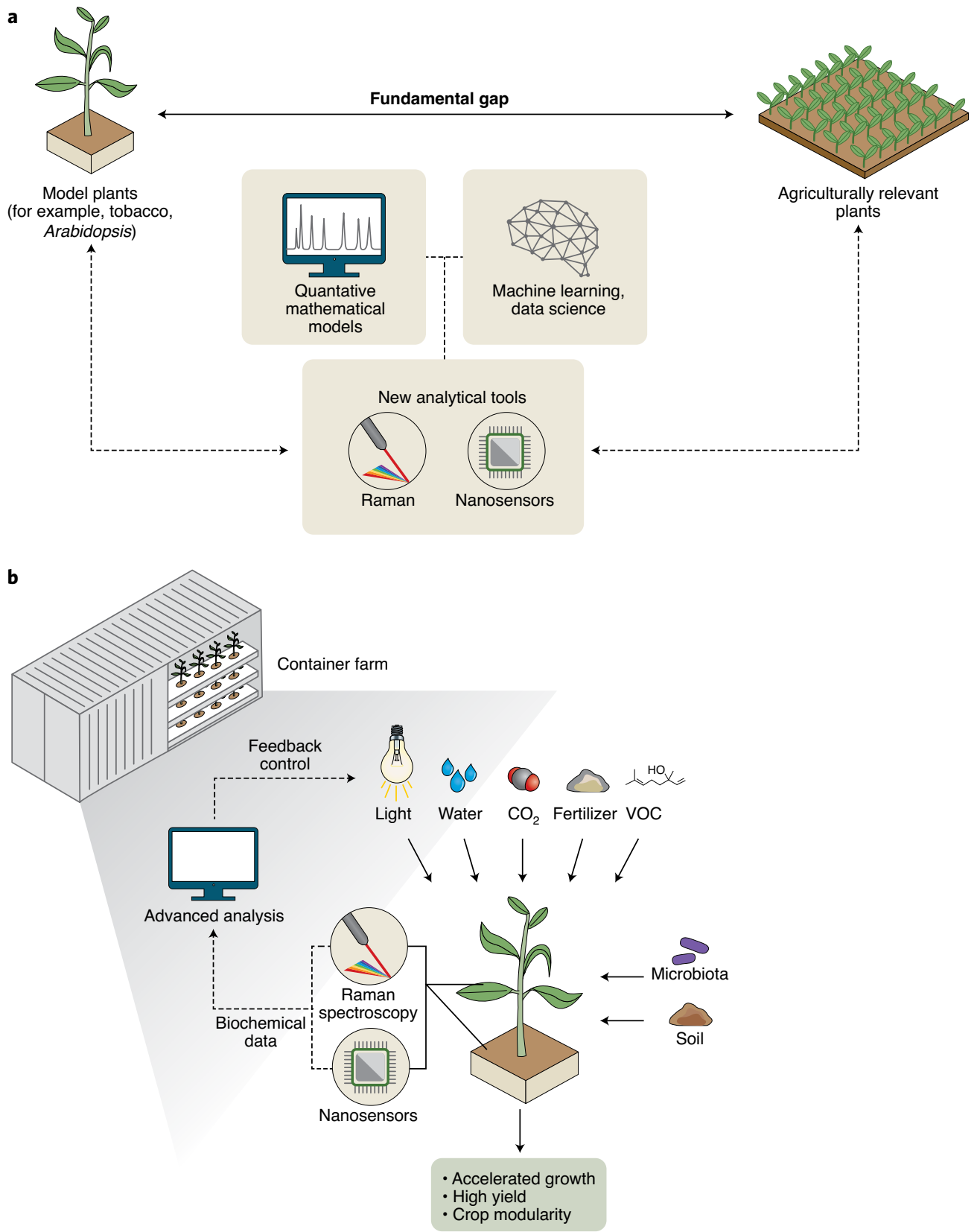

Fig. 2 | Application of species-independent diagnostic tools in next-generation agriculture. a, Bridging the gap between model plants and agriculturally relevant crops requires new analytical tools in conjunction with computational resources, including machine learning and advanced data analytics. $\mathbf{b}$, Feedback control concept for high-density agriculture enabled by next-generation diagnostic tools.

Machine-learning techniques and mathematical models, both formulated based on the information gathered by species-agnostic tools, can potentially allow the extension of findings across species (Fig. 2a). This can yield new insights previously inaccessible through traditional plant biology approaches. For example, the speed and characteristics of a wound-induced $\mathrm{H}_{2} \mathrm{O}_{2}$ wave probed by nanosensors appeared to be controlled by the antioxidant capacity of different plant species ${ }^{76}$. Machine-learning algorithms can be similarly applied to the readings obtained by nanosensors or spectroscopy tools to predict plant behaviour across species ${ }^{107}$.

Another potential opportunity of these newly developed tools is afforded by their ability to probe systemic signalling within the plants, often in real time. As these technologies are non-destructive and can be used to extract information from the plants at a whole-plant resolution, we can study how local stimuli induce the propagation of signals from one organ or organelle to another within the same plant, organ or cell. Such investigations can reveal many of the underlying mechanisms that mediate systemic signalling ${ }^{108}$. These tools also enable the same organ or cell to be studied over time, circumventing the problem of plant-to-plant variations in data collection and analysis ${ }^{109}$. Studying the dynamics of phytohormones in non-model plants with nanosensors can provide insights into how these plants grow and adapt in agricultural settings. Their temporal and spatial profile can be monitored to report the onset of resource deficiency and stresses. These phytohormones often exist in low, transient concentrations and vary according to spatial locations within the plant. Furthermore, there are often various isomers of phytohormones, rendering their detection via 
conventional methods, such as analysis by high-performance liquid chromatography/gas chromatography and serological assays, difficult ${ }^{100}$. By contrast, field-deployable miniaturized sensors could be cost-effective diagnostic tools to monitor plant health and development. The use of engineered nanoparticles, located within the plant tissues and subcellular compartments, may enable biochemical pathways to be studied selectively with a greater precision and at a shorter timescale than existing approaches ${ }^{71,110}$. Decoding plant signalling pathways non-destructively for agriculturally important plants as well as model systems ubiquitously explored in plant biology will lead to radically new agricultural technologies: from rapid plant phenotyping to early diagnostics of plant health status.

In addition, the physical and chemical properties of nanoparticles can also be engineered to tune their localization within plant cells. Nanoparticles with dimensions below the size exclusion limit of the cell wall $(\sim 20-50 \mathrm{~nm})$ have the ability to access the plant cell interior ${ }^{111}$. It has been shown that the zeta potential and size of nanoparticles govern their subcellular localization within plant cells, enabling the specific targeting of various compartments such as the chloroplasts and the cytosol ${ }^{75,112,113}$. Furthermore, targeting moieties can be grafted onto the surface of nanostructures to direct their localization to the nucleus, mitochondria or other organelles within a plant cell ${ }^{114,115}$. While these strategies can be rationally employed to control nanoparticle transport into specific organelles, biomolecules present in the plant biological environment can rapidly adsorb onto nanoparticle surfaces in an energetically favourable process and alter the nanoparticle surface characteristics ${ }^{116,117}$. The new corona formation due to adsorbed proteins can unpredictably change nanoparticle identity and localization ${ }^{118,119}$. The interaction between proteins and other biomolecules on nanoparticle corona formation in the plant biological environment needs to be further investigated. The targeted delivery of nanosensors to different plant organelles will help elucidate the subcellular dynamics of signalling molecules within model plants as well as agriculturally relevant crops. The ability to precisely deliver nanosensors to specific plant compartments can also enable multiplexing of different molecules to help untangle the complex interplay of plant signalling pathways.

In addition to monitoring the phytohormones and/or other growth-promoting metabolites, we also propose a holistic approach to apply multi-dimensional spectral analyses to the phytobiome and the soil or rhizospheric microbiome, both of which are important contributors to plant phenotypes and crop yields. The crop yield is a complex phenotype, and such tripartite interactions that consider plant metabolism, soil and microbiota not only influence proper growth and development in plants, but also the overall health and metabolic resilience. Microbiota associated with the rhizosphere and phyllosphere contribute significantly to plant growth and to tolerance or resistance to biotic and abiotic stresses ${ }^{120-122}$. Probing the plant-associated microbiome community can provide a wealth of information about the crop development and health ${ }^{123-125}$. The fluorescent nanosensors described in the previous section can be easily interfaced within a fibre optic platform, creating an electrode-like or optode probe for the detection of local nutrients, pollutants and specific microorganisms in the soil. In this configuration, nanosensors are mounted at the tip of an optical fibre which performs both the excitation and signal collection for fluorescence monitoring, as we and others have shown recently ${ }^{126,127}$. The nanosensor-optode form factor has a high mechanical flexibility and can be compactly integrated into a portable detection platform that can utilize the same hardware as Raman spectroscopy. This presents a promising approach for multi-dimensional spectral monitoring of soil in the field. We anticipate that Raman microspectroscopy and imaging will prove to be useful tools to identify chemical signatures and soil constituents $^{128}$ associated with the beneficial phytobiome systems that promote plant growth. In addition, a portable fluorescence imaging system has been developed for automated phenotyping of root architecture in the field ${ }^{129}$. The analytical technology platforms highlighted here could be leveraged to probe ongoing soil health, phenotype root architecture directly and elucidate beneficial microbial traits. Non-invasive soil phenotyping allows plant and microbial traits to be measured on the same population over time, and offers opportunities for simultaneous monitoring of shoot and root growth ${ }^{130}$. Such an approach may help identify and utilize the critical growth-promoting metabolites as well as the crucial metabolic hubs towards improved crop yield and resilience ${ }^{131}$. Likewise, surface-enhanced Raman spectroscopy could be applied in conjunction with detailed metabolomics to identify the molecular and chemical basis of plant-soil-microbiome interactions ${ }^{132,133}$, thus enabling the development of knowledge-based intelligent solutions for precision agriculture.

An effective implementation of nanosensors and spectroscopy platforms in the field will require a concomitant development in low-cost and high-throughput image analysis tools for plant phenotyping. Such tools can be used to interpret the sensor readings with high quantitative and temporal resolution. For example, the recently introduced PlantCV, an open-source image analysis software for plant phenotyping, can correlate plant phenotypes with stress treatments using low-cost electronics in an automated fashion $^{134}$. By coupling advances in image analysis algorithms with new sensor technologies, we can extract information about not just the visible plant phenotype but also the internal metabolite dynamics for early diagnosis. Recent demonstrations that optical signals from nanosensors can be read out with a Raspberry Pi platform highlight the potential ability of these promising technologies to integrate with existing portable platforms ${ }^{76,77}$. Indeed, coupling remote sensing technologies with micro or nanosensors which are easy to use and able to provide the required precision would be key to making remote sensor platforms useful. Developing an integrated platform that combines species-agnostic tools with advanced imaging platforms and data analysis packages will enable a large-scale field data collection at high spatial and temporal resolutions for non-destructive plant phenotyping and early diagnostics. While some progress has been made in demonstrating the development and utility of these tools, successfully integrating all the different components into an integrated platform that is reliable, affordable and accessible for end-users remains a fundamental challenge.

\section{New analytics to advance urban farming}

As a product of agriculture, the plant is itself a highly complex machine within a dynamic ecosystem. The fundamental study of its internal workings, hydraulics, chemical signalling and genetic pathways remains an important and emerging scientific enterprise. Moreover, there are diverse microbial communities in the soil and plant tissues which promote plant development through improved nutrient acquisition, accelerated growth and increased host resistance against stresses ${ }^{135,136}$. Handheld Raman spectrometers that allow a form of laser-induced, biochemical fingerprinting of living plants yield access to the internal workings of plants with unprecedented precision. Similarly, nanotechnology-enabled molecular recognition allows measurement of plant signalling hormones and stress responses in a way that can 'decode' the inner communication within the plant itself. They can also be employed to characterize rhizospheric and phyllospheric microbiota. Such modern tools and related technologies may open possibilities for long sought-after feedback control schemes to accelerate and improve plant growth, yield, nutrition and culinary properties in urban farm settings (Fig. 2b). Water, light and fertilizer are all example inputs whose amounts and applied durations can be digitally controlled subject to internal responses within the plant itself. The result can be optimal growth conditions for potentially any plant, as well as the seamless transition from one crop to another in successive growth cycles. 
This accelerated productivity combined with extreme modularity in farming could be highly enabling for next-generation urban farming.

An opportunity for advanced analytics that is important in the context of high-density farming, and urban farming in particular, is in addressing the shade avoidance syndrome (SAS). This adaptive response in plants is triggered to overcome shade conditions caused by surrounding vegetation and includes stem and petiole elongation, hyponastic leaves, reduced leaf development, early flowering and increased senescence ${ }^{137}$. The phenotypic and physiological changes of SAS are detrimental to agriculture, and specifically urban farming due to its irreversibility ${ }^{138,139}$. Due to the morphological plasticity of plants during SAS, early detection and real-time monitoring of SAS are crucial for sustainable agriculture. Advanced analytical tools, such as Raman spectroscopy and nanosensor technology, could augment or replace conventional assessments of SAS, which are mostly restricted to the genetic level and the gene expression of shade-induced genes (AtHB2, AtHB4, PIL1 and so on $)^{140-142}$.

\section{Technological status and economic potential of advanced analytical tools}

Biotic and abiotic stresses cause substantial losses in crop yield and the economic value of agricultural products. Plant diseases and pests cause an estimated average yield loss of $30.0 \%$ in rice, $22.6 \%$ in maize, $21.5 \%$ in wheat, $21.4 \%$ in soybean and $17.2 \%$ in potato worldwide ${ }^{6}$. Global wheat production is predicted to decrease by $6 \%$ for each degree Celsius rise in temperature ${ }^{143}$ Drought alone is responsible for more substantial crop losses annually than all pathogens combined, with approximately $\$ 30$ billion worth of global losses in crop production over the past decade ${ }^{5}$. The severity and frequency of these stresses on crop production are further exacerbated by climate change ${ }^{144-146}$. Advanced analytical tools highlighted here can help offset the economic losses caused by these factors through early stress detection in crops. However, to facilitate widespread adoption of these technologies in agriculture, their economic potential and reliability need to be validated to ensure that they remain affordable and more effective than the existing approaches.

The economics of deploying species-independent platforms in agriculture depends on a number of factors such as the type of agriculture (for example, open-field agriculture or urban farms), crop selection (high-value produce or more commodity staples) as well as the nature of the analytical tools employed. Here, we illustrate some cases whereby the use of sensors may be economically advantageous. The profitability of portable Raman spectroscopy for precision agriculture applications can be modelled on previous work studying the economic benefits of portable reflectance analysers for inferring crop yield and nitrogen demand. Portable reflectance sensors (for example, Greenseeker) have been used to guide the variable rate application (VRA) of nitrogen fertilizer. Previous studies have demonstrated a value for precision delivery of nitrogen fertilizers to be $\$ 16-29 \mathrm{ha}^{-1}$ for a commodity crop such as wheat ${ }^{147}$. In practice, reflectance analysis is an imperfect assay for plant nitrogen, as the reflectance also changes with temperature, water availability and time within the growing season. By contrast, Raman spectroscopy can be employed to specifically measure nitrogen status in plants ${ }^{94}$. Today, a portable Raman analyser may cost approximately $\$ 12,000$. If a consulting engineering firm were to provide services at the cost of their technical labour $\left(\$ 16 \mathrm{~h}^{-1}\right)$ and capital depreciation (4-yr depreciation, $300 \mathrm{~h}$ annually for measurements with $20 \%$ residual value), Raman analysis would cost $\$ 8 \mathrm{ha}^{-1}$, assuming $15 \mathrm{~min}$ of operation per hectare (10 min of walking and 5 min of Raman acquisition). The economic benefit (\$16-29 ha-1) relative to the cost $\left(\$ 12 \mathrm{ha}^{-1}\right)$ is significant, even for a commodity such as wheat and assuming a utilization of only $300 \mathrm{~h}$ annually.
In practice, higher value crops such as vegetables and more demanding schedules ( $900 \mathrm{~h}$ annually) might be more reflective of the margins for a mature business employing Raman spectroscopy for precision agriculture. Within the urban farming context, the use of non-destructive sensors has allowed companies such as AeroFarms to apply fertilizers frequently and in small doses, adjusting along the way to optimize plant growth. Such adjustments may be made as frequently as 15 -min intervals, coupled with sensors which tightly control humidity and water consumption, driving cost efficiencies in water and fertilizer use. The fluorescence of optical nanosensors can also be monitored with a Raman spectrometer ${ }^{148}$. As such, the same portable instrument can be used to obtain both the Raman spectra and the nanosensor information at no extra cost. Furthermore, advanced analytical tools not only drive efficiencies from the cost perspective, such as reduced fertilizer application, but they can also benefit the revenue side from increased product quality. For instance, sensors that can detect optimal harvesting conditions to tune the flavour profiles of crops ${ }^{149-151}$ may translate into products that consumers are willing to pay a price premium for, further enhancing the value proposition for targeted sensor deployment within different agricultural settings.

In addition to profitability, the technological robustness and sensitivity of these potentially disruptive technologies need to be further evaluated for field applications. While some of the described tools have been shown to work in laboratory or greenhouse conditions $s^{76,77,94,102}$, the complex and constantly changing environment in the field poses a different set of challenges and opportunities to validate the performance of these technologies. For example, the stability, sensitivity and selectivity of nanosensors may be affected by weather, plant developmental stage and soil types. Plant scientists and researchers have an important role to validate the performance of these tools under variable conditions in the laboratory in parallel to their application in the field. The variability in adaptive responses between plant species and subspecies to stresses, the incidence of multiple stresses as well as the biodiversity of pathogens, insects and microbes further add to complexity of agricultural systems in which the reliability of species-independent tools needs to be assessed ${ }^{152,153}$. Multiplexing different sensors and analytical tools can help increase the precision of crop diagnostics, but this will increase the operational cost for growers, and the optimal combinations may vary from one field to another. Systems-level evaluations can be useful to identify the optimal design space in which the accuracy and economic impact of the deployable platforms can be maximized ${ }^{154}$. The impact of different soil characteristics and climate conditions on the linearity and robustness of the proposed tools can be assessed through large-scale field data collection at geographically diverse sites. Data from such studies can also inform further redesign and refinement of these technologies to ensure their effective deployment in agriculture ${ }^{155}$

With these new tools, questions arise as to how many sensors must be deployed to monitor multiple hectares of plants and at what frequency. In the absence of any additional information, except that such plants follow a distribution in desired traits or status, the problem is one for basic statistics. However, this neglects the prior information that researchers will collect though the steady deployment and data collection using these tools. Mathematical models and machine learning that leverage data analytics form an essential part of the sensors themselves. When combined, new opportunities will likely emerge. The monitoring of a much smaller cohort of plants could inform the field. The concept of strategically placed 'sentinel plants' can dramatically reduce the necessary deployment scale ${ }^{156,157}$. Such an approach can be complimented by the use of drone technology to check and update such models without the burden of scanning the entire plantation ${ }^{158,159}$. In contrast, the urban farm does not have the same challenges with geographical scale, but will benefit from the same overall approach. 


\section{Conclusion and outlook}

This Perspective highlights that we now have first-generation examples and promising concepts of how a new generation of tools can revolutionize plant science and agriculture. The development of sensors and non-invasive technology to study plant signalling and physiology in situ would now allow us to extend investigations normally conducted under laboratory conditions to field-grown crops, making use of the knowledge base accumulated using model plants such as Arabidopsis, rice and tomato. With the advent of advanced analytical tools, the challenge becomes the translation of the raw data collected into meaningful information for the farmers to make profitable decisions. The addition of Internet of Things (IoT) to sensors, big data analysis and incorporation of artificial intelligence techniques will promote data-driven agriculture to enable sustainable farming ${ }^{160}$. Furthermore, for these species-independent platforms to be accessible and affordable to agricultural end-users, there must be an increased collaboration among engineers, data scientists and plant biologists. There has to be a change in paradigm and mind-set that these fields are separate to encourage more collaboration between these different groups. Sustained engagement with the public, farmers and plant breeding programmes can help accelerate the acceptance and integration of these tools in everyday farming practices. It is paramount that more testing of species-independent tools is conducted under field conditions, with critical evaluations of their technical robustness and economic potential to ensure sustainable implementation of these technologies in tomorrow's agriculture.

Received: 30 April 2020; Accepted: 16 October 2020;

Published online: 30 November 2020

\section{References}

1. Godfray, H. C. J. et al. Food security: the challenge of feeding 9 billion people. Science 327, 812-818 (2010).

2. Gerten, D. et al. Feeding ten billion people is possible within four terrestrial planetary boundaries. Nat. Sustain. 3, 200-208 (2020).

3. King, T. et al. Food safety for food security: relationship between global megatrends and developments in food safety. Trends Food Sci. Tech. 68, 160-175 (2017).

4. Suzuki, N., Rivero, R. M., Shulaev, V., Blumwald, E. \& Mittler, R. Abiotic and biotic stress combinations. New Phytol. 203, 32-43 (2014).

5. Gupta, A., Rico-Medina, A. \& Caño-Delgado, A. I. The physiology of plant responses to drought. Science 368, 266-269 (2020).

6. Savary, S. et al. The global burden of pathogens and pests on major food crops. Nat. Ecol. Evol. 3, 430-439 (2019).

7. van Esse, H. P., Reuber, T. L. \& van der Does, D. Genetic modification to improve disease resistance in crops. New Phytol. 225, 70-86 (2020).

8. Lesk, C., Rowhani, P. \& Ramankutty, N. Influence of extreme weather disasters on global crop production. Nature 529, 84-87 (2016).

9. Dai, A. Increasing drought under global warming in observations and models. Nat. Clim. Change 3, 52-58 (2013).

10. van Meijl, $\mathrm{H}$. et al. Comparing impacts of climate change and mitigation on global agriculture by 2050. Environ. Res. Lett. 13, 064021 (2018).

11. Wiebe, K., Robinson, S. \& Cattaneo, A. in Sustainable Food and Agriculture 55-74 (Elsevier, 2019).

12. Walter, A., Finger, R., Huber, R. \& Buchmann, N. Smart farming is key to developing sustainable agriculture. Proc. Natl Acad. Sci. USA 114, 6148-6150 (2017).

13. Li, S. et al. Modulating plant growth-metabolism coordination for sustainable agriculture. Nature 560, 595-600 (2018).

14. Balmford, A. et al. The environmental costs and benefits of high-yield farming. Nat. Sustain. 1, 477-485 (2018).

15. Specht, K. et al. Urban agriculture of the future: an overview of sustainability aspects of food production in and on buildings. Agric. Human Values 31, 33-51 (2014).

16. Benke, K. \& Tomkins, B. Future food-production systems: vertical farming and controlled-environment agriculture. Sustain. Sci. Pract. Policy 13, 13-26 (2017).

17. Torero Cullen, M. A battle plan for ensuring global food supplies during the COVID-19 crisis. FAO http://www.fao.org/news/story/en/item/1268059/ icode/ (2020).

18. Berens, M. L. et al. Balancing trade-offs between biotic and abiotic stress responses through leaf age-dependent variation in stress hormone cross-talk. Proc. Natl Acad. Sci. USA 116, 2364-2373 (2019).
19. Riedlmeier, M. et al. Monoterpenes support systemic acquired resistance within and between plants. Plant Cell 29, 1440-1459 (2017).

20. Zhu, J. K. Abiotic stress signaling and responses in plants. Cell 167, 313-324 (2016)

21. Rhee, S. Y. et al. The Arabidopsis Information Resource (TAIR): a model organism database providing a centralized, curated gateway to Arabidopsis biology, research materials and community. Nucleic Acids Res. 31, 224-228 (2003).

22. Bevan, M. \& Walsh, S. The Arabidopsis genome: a foundation for plant research. Genome Res. 15, 1632-1642 (2005).

23. Li, Z. \& Sillanpää, M. J. Dynamic quantitative trait locus analysis of plant phenomic data. Trends Plant Sci. 20, 822-833 (2015).

24. Walia, A., Waadt, R. \& Jones, A. M. Genetically encoded biosensors in plants: pathways to discovery. Annu. Rev. Plant Biol. 69, 497-524 (2018).

25. Borrill, P. Blurring the boundaries between cereal crops and model plants. New Phytol. 9, 16229 (2019).

26. Carpentler, S. C. et al. Proteome analysis of non-model plants: a challenging but powerful approach. Mass Spectrom. Rev. 27, 354-377 (2008).

27. Long, S. P., Marshall-Colon, A. \& Zhu, X. G. Meeting the global food demand of the future by engineering crop photosynthesis and yield potential. Cell 161, 56-66 (2015).

28. Jackson, S. A. Rice: the first crop genome. Rice 9, 1-3 (2016).

29. Yang, N. et al. Genome assembly of a tropical maize inbred line provides insights into structural variation and crop improvement. Nat. Genet. 51, 1052-1059 (2019).

30. Sant'Ana, D. V. P. \& Lefsrud, M. Tomato proteomics: tomato as a model for crop proteomics. Scientia Horticulturae 239, 224-233 (2018).

31. Unamba, C. I. N., Nag, A. \& Sharma, R. K. Next generation sequencing technologies: the doorway to the unexplored genomics of non-model plants. Front. Plant Sci. 6, 1074 (2015).

32. Richards, C. L. et al. Ecological plant epigenetics: evidence from model and non-model species, and the way forward. Ecol. Lett. 20, 1576-1590 (2017).

33. Lisec, J., Schauer, N., Kopka, J., Willmitzer, L. \& Fernie, A. R. Gas chromatography mass spectrometry-based metabolite profiling in plants. Nat. Protoc. 1, 387-396 (2006).

34. Fernie, A. R., Trethewey, R. N., Krotzky, A. J. \& Willmitzer, L. Metabolite profiling: from diagnostics to systems biology. Nat. Rev. Mol. Cell Biol. 5, 763-769 (2004).

35. Lytovchenko, A. et al. Application of GC-MS for the detection of lipophilic compounds in diverse plant tissues. Plant Methods 5, 4 (2009).

36. Nagano, A. J. et al. Deciphering and prediction of transcriptome dynamics under fluctuating field conditions. Cell 151, 1358-1369 (2012).

37. Shakoor, N., Lee, S. \& Mockler, T. C. High throughput phenotyping to accelerate crop breeding and monitoring of diseases in the field. Curr. Opin. Plant Biol. 38, 184-192 (2017).

38. Araus, J. L. \& Cairns, J. E. Field high-throughput phenotyping: the new crop breeding frontier. Trends Plant Sci. 19, 52-61 (2014).

39. Kirchgessner, N. et al. The ETH field phenotyping platform FIP: a cable-suspended multi-sensor system. Funct. Plant Biol. 44, 154 (2017).

40. Pieruschka, R. \& Schurr, U. Plant phenotyping: past, present, and future. Plant Phenomics 2019, 7507131 (2019).

41. Yang, G. et al. Unmanned aerial vehicle remote sensing for field-based crop phenotyping: current status and perspectives. Front. Plant Sci. 8, 1111 (2017).

42. Ampatzidis, Y. \& Partel, V. UAV-based high throughput phenotyping in citrus utilizing multispectral imaging and artificial intelligence. Remote Sens. 11, 410 (2019).

43. Li, L., Zhang, Q. \& Huang, D. A review of imaging techniques for plant phenotyping. Sensors 14, 20078-20111 (2014).

44. Chawade, A. et al. High-throughput field-phenotyping tools for plant breeding and precision agriculture. Agronomy 9, 258 (2019).

45. Zhao, C. et al. Crop phenomics: current status and perspectives. Front. Plant Sci. 10, 714 (2019).

46. Bai, G., Ge, Y., Hussain, W., Baenziger, P. S. \& Graef, G. A multi-sensor system for high throughput field phenotyping in soybean and wheat breeding. Comput. Electron. Agric. 128, 181-192 (2016).

47. Cohen, Y., Alchanatis, V., Meron, M., Saranga, Y. \& Tsipris, J. Estimation of leaf water potential by thermal imagery and spatial analysis. J. Exp. Bot. 56, 1843-1852 (2005).

48. Valle, B. et al. PYM: a new, affordable, image-based method using a Raspberry Pi to phenotype plant leaf area in a wide diversity of environments. Plant Methods 13, 1-17 (2017).

49. Gerhards, M., Schlerf, M., Mallick, K. \& Udelhoven, T. Challenges and future perspectives of multi-/hyperspectral thermal infrared remote sensing for crop water-stress detection: a review. Remote Sens. 11, 1240 (2019).

50. Yang, W. et al. Crop phenomics and high-throughput phenotyping: past decades, current challenges, and future perspectives. Mol. Plant 13, 187-214 (2020). 
51. Fukatsu, T., Watanabe, T., Hu, H., Yoichi, H. \& Hirafuji, M. Field monitoring support system for the occurrence of Leptocorisa chinensis Dallas (Hemiptera: Alydidae) using synthetic attractants, Field Servers, and image analysis. Comput. Electron. Agric. 80, 8-16 (2012).

52. Sadeghi-Tehran, P., Sabermanesh, K., Virlet, N. \& Hawkesford, M. J. Automated method to determine two critical growth stages of wheat: heading and flowering. Front. Plant Sci. 8, 252 (2017).

53. Duan, L. et al. Novel digital features discriminate between drought resistant and drought sensitive rice under controlled and field conditions. Front. Plant Sci. 9, 492 (2018)

54. Jahnke, S. et al. phenoSeeder - a robot system for automated handling and phenotyping of individual seeds. Plant Physiol. 172, 1358-1370 (2016).

55. Mahlein, A. K. Plant disease detection by imaging sensors - parallels and specific demands for precision agriculture and plant phenotyping. Plant Dis. 100, 241-254 (2016).

56. Alves Varella, C. A., Gleriani, J. M. \& dos Santos, R. M. in Sugarcane: Agricultural Production, Bioenergy and Ethanol 185-203 (Elsevier Inc., 2015).

57. Tantalaki, N., Souravlas, S. \& Roumeliotis, M. Data-driven decision making in precision agriculture: the rise of big data in agricultural systems. J. Agric. Food Inf. 20, 344-380 (2019).

58. Baumgart-Getz, A., Prokopy, L. S. \& Floress, K. Why farmers adopt best management practice in the United States: a meta-analysis of the adoption literature. J. Environ. Manage. 96, 17-25 (2012).

59. Fujita, M. et al. Crosstalk between abiotic and biotic stress responses: a current view from the points of convergence in the stress signaling networks. Curr. Opin. Plant Biol. 9, 436-442 (2006).

60. Perilla-Henao, L. M. \& Casteel, C. L. Vector-borne bacterial plant pathogens: interactions with hemipteran insects and plants. Front. Plant Sci. 7, 1163 (2016)

61. Bendix, C. \& Lewis, J. D. The enemy within: phloem-limited pathogens. Mol. Plant Pathol. 19, 238-254 (2018).

62. Choi, W.-G. et al. Orchestrating rapid long-distance signaling in plants with $\mathrm{Ca}^{2+}$, ROS and electrical signals. Plant J. 90, 698-707 (2017).

63. Gilroy, S. et al. ROS, calcium, and electric signals: key mediators of rapid systemic signaling in plants. Plant Physiol. 171, 1606-1615 (2016)

64. Shan, X., Yan, J. \& Xie, D. Comparison of phytohormone signaling mechanisms. Curr. Opin. Plant Biol. 15, 84-91 (2012).

65. Zhao, Y., Qi, Z. \& Berkowitz, G. A. Teaching an old hormone new tricks: cytosolic $\mathrm{Ca}^{2+}$ elevation involvement in plant brassinosteroid signal transduction cascades. Plant Physiol. 163, 555-565 (2013).

66. Seyfferth, C. \& Tsuda, K. Salicylic acid signal transduction: the initiation of biosynthesis, perception and transcriptional reprogramming. Front. Plant Sci. 5, 697 (2014)

67. Mohanta, T. K., Kumar, P. \& Bae, H. Genomics and evolutionary aspect of calcium signaling event in calmodulin and calmodulin-like proteins in plants. BMC Plant Biol. 17, 38 (2017).

68. Huang, H., Ullah, F., Zhou, D. X., Yi, M. \& Zhao, Y. Mechanisms of ROS regulation of plant development and stress responses. Front. Plant Sci. 10, 800 (2019).

69. Wang, C., Liu, Y., Li, S. S. \& Han, G. Z. Insights into the origin and evolution of the plant hormone signaling machinery. Plant Physiol. 167, 872-886 (2015).

70. Blázquez, M. A., Nelson, D. C. \& Weijers, D. Evolution of plant hormone response pathways. Annu. Rev. Plant Biol. 71, 327-353 (2020).

71. Lew, T. T. S., Koman, V. B., Gordiichuk, P., Park, M. \& Strano, M. S. The emergence of plant nanobionics and living plants as technology. Adv. Mater. Technol. 5, 1900657 (2020).

72. Giraldo, J. P., Wu, H., Newkirk, G. M. \& Kruss, S. Nanobiotechnology approaches for engineering smart plant sensors. Nat. Nanotechnol. 14, 541-553 (2019).

73. Kwak, S. Y. et al. Nanosensor technology applied to living plant systems. Annu. Rev. Anal. Chem. 10, 113-140 (2017).

74. Zhu, C., Yang, G., Li, H., Du, D. \& Lin, Y. Electrochemical sensors and biosensors based on nanomaterials and nanostructures. Anal. Chem. 87, 230-249 (2015).

75. Kwak, S. Y. et al. Chloroplast-selective gene delivery and expression in planta using chitosan-complexed single-walled carbon nanotube carriers. Nat. Nanotechnol. 14, 447-455 (2019).

76. Lew, T. T. S. et al. Real-time detection of wound-induced $\mathrm{H}_{2} \mathrm{O}_{2}$ signalling waves in plants with optical nanosensors. Nat. Plants 6, 404-415 (2020).

77. Wong, M. H. et al. Nitroaromatic detection and infrared communication from wild-type plants using plant nanobionics. Nat. Mater. 16, 264-272 (2017).

78. Zrazhevskiy, P., Sena, M. \& Gao, X. Designing multifunctional quantum dots for bioimaging, detection, and drug delivery. Chem. Soc. Rev. 39 4326-4354 (2010).

79. Li, J., Wu, H., Santana, I., Fahlgren, M. \& Giraldo, J. P. Standoff optical glucose sensing in photosynthetic organisms by a quantum dot fluorescent probe. ACS Appl. Mater. Interfaces 10, 28279-28289 (2018).
80. Li, W. et al. Phytotoxicity, uptake, and translocation of fluorescent carbon dots in mung bean plants. ACS Appl. Mater. Interfaces 8, 19939-19945 (2016).

81. Yao, Z., Lai, Z., Chen, C., Xiao, S. \& Yang, P. Full-color emissive carbon-dots targeting cell walls of onion for in situ imaging of heavy metal pollution. Analyst 144, 3685 (2019).

82. Hasegawa, Y., Murohashi, F. \& Uchida, H. Plant physiological activity sensing by bioelectric potential measurement. Procedia Engineer. 168 630-633 (2016)

83. Ochiai, T., Tago, S., Hayashi, M. \& Fujishima, A. Highly sensitive measurement of bio-electric potentials by boron-doped diamond (BDD) electrodes for plant monitoring. Sensors 15, 26921-26928 (2015).

84. Tenenboim, H. \& Brotman, Y. Omic relief for the biotically stressed: metabolomics of plant biotic interactions. Trends Plant Sci. 21, 781-791 (2016).

85. Kushalappa, A. C. \& Gunnaiah, R. Metabolo-proteomics to discover plant biotic stress resistance genes. Trends Plant Sci. 18, 522-531 (2013).

86. Jorge, T. F. et al. Mass spectrometry-based plant metabolomics: metabolite responses to abiotic stress. Mass Spectrom. Rev. 35, 620-649 (2016).

87. Obata, T. \& Fernie, A. R. The use of metabolomics to dissect plant responses to abiotic stresses. Cell. Mol. Life Sci. 69, 3225-3243 (2012).

88. Ward, J. L. et al. The metabolic transition during disease following infection of Arabidopsis thaliana by Pseudomonas syringae pv. tomato. Plant J. 63, 443-457 (2010).

89. Gunnaiah, R., Kushalappa, A. C., Duggavathi, R., Fox, S. \& Somers, D. J. Integrated metabolo-proteomic approach to decipher the mechanisms by which wheat qtl (Fhb1) contributes to resistance against Fusarium graminearum. PLoS ONE 7, e40695 (2012).

90. Kim, H. K., Choi, Y. H. \& Verpoorte, R. NMR-based plant metabolomics: where do we stand, where do we go? Trends Biotechnol. 29, 267-275 (2011).

91. Cevallos-Cevallos, J. M., Futch, D. B., Shilts, T., Folimonova, S. Y. \& Reyes-De-Corcuera, J. I. GC-MS metabolomic differentiation of selected citrus varieties with different sensitivity to citrus huanglongbing. Plant Physiol. Biochem. 53, 69-76 (2012).

92. Altangerel, N. et al. In vivo diagnostics of early abiotic plant stress response via Raman spectroscopy. Proc. Natl Acad. Sci. USA 114, 3393-3396 (2017).

93. Yeturu, S. et al. Handheld Raman spectroscopy for the early detection of plant diseases: Abutilon mosaic virus infecting Abutilon sp. Anal. Methods 8, 3450-3457 (2016).

94. Hao Huang, C. et al. Early diagnosis and management of nitrogen deficiency in plants utilizing Raman spectroscopy. Front. Plant Sci. 11, 663 (2020).

95. Farber, C., Mahnke, M., Sanchez, L. \& Kurouski, D. Advanced spectroscopic techniques for plant disease diagnostics. A review. Trends Analyt. Chem. 118, 43-49 (2019).

96. Angel, S. M., Gomer, N. R., Sharma, S. K. \& McKay, C. Remote Raman spectroscopy for planetary exploration: a review. Appl. Spectrosc. 66, 137-50 (2012).

97. Acosta-Maeda, T. E. et al. Remote Raman measurements of minerals, organics, and inorganics at $430 \mathrm{~m}$ range. Appl. Opt. 55, 10283 (2016).

98. Dudareva, N., Negre, F., Nagegowda, D. A. \& Orlova, I. Plant volatiles: recent advances and future perspectives. CRC. Crit. Rev. Plant Sci. 25, 417-440 (2006).

99. Vivaldo, G., Masi, E., Taiti, C., Caldarelli, G. \& Mancuso, S. The network of plants volatile organic compounds. Sci. Rep. 7, 11050 (2017).

100. Martinelli, F. et al. Advanced methods of plant disease detection. A review. Agro. Sustain. Dev. 35, 1-25 (2015).

101. Tholl, D. et al. Practical approaches to plant volatile analysis. Plant J. 45, 540-560 (2006).

102. Li, Z. et al. Non-invasive plant disease diagnostics enabled by smartphonebased fingerprinting of leaf volatiles. Nat. Plants 5, 856-866 (2019).

103. Fong, D., Luo, S. X., Andre, R. S. \& Swager, T. M. Trace ethylene sensing via wacker oxidation. ACS Cent. Sci. 6, 507-512 (2020).

104. Cui, S., Ling, P., Zhu, H. \& Keener, H. Plant pest detection using an artificial nose system: a review. Sensors 18, 378 (2018).

105. Zhang, Y. et al. Efficient and transgene-free genome editing in wheat through transient expression of CRISPR/Cas9 DNA or RNA. Nat. Commun. 7, 667-697 (2016)

106. Chen, K., Wang, Y., Zhang, R., Zhang, H. \& Gao, C. CRISPR/Cas genome editing and precision plant breeding in agriculture. Annu. Rev. Plant Biol. 70, 667-697 (2019).

107. Singh, A. K., Ganapathysubramanian, B., Sarkar, S. \& Singh, A. Deep learning for plant stress phenotyping: trends and future perspectives. Trends Plant Sci. 23, 883-898 (2018).

108. Fichman, Y., Miller, G. \& Mittler, R. Whole-plant live imaging of reactive oxygen species. Mol. Plant 12, 1203-1210 (2019).

109. Dhondt, S., Wuyts, N. \& Inzé, D. Cell to whole-plant phenotyping: the best is yet to come. Trends Plant Sci. 18, 428-439 (2013).

110. Giraldo, J. P. et al. Plant nanobionics approach to augment photosynthesis and biochemical sensing. Nat. Mater. 13, 400-408 (2014) 
111. Cunningham, F. J., Goh, N. S., Demirer, G. S., Matos, J. L. \& Landry, M. P. Nanoparticle-mediated delivery towards advancing plant genetic engineering. Trends Biotechnol. 36, 882-897 (2018).

112. Lew, T. T. S. et al. Rational design principles for the transport and subcellular distribution of nanomaterials into plant protoplasts. Small 14, 1802086 (2018).

113. Lew, T. T. S. et al. Nanocarriers for transgene expression in pollen as a plant biotechnology tool. ACS Mater. Lett. 2, 1057-1066 (2020).

114. Thagun, C., Chuah, J. \& Numata, K. Targeted gene delivery into various plastids mediated by clustered cell-penetrating and chloroplast-targeting peptides. Adv. Sci. 6, 1902064 (2019).

115. Yoshizumi, T., Oikawa, K., Chuah, J. A., Kodama, Y. \& Numata, K. Selective gene delivery for integrating exogenous DNA into plastid and mitochondrial genomes using peptide-DNA complexes. Biomacromolecules 19, 1582-1591 (2018).

116. Hadjidemetriou, M. \& Kostarelos, K. Nanomedicine: evolution of the nanoparticle corona. Nat. Nanotechnol. 12, 288-290 (2017).

117. Zhdanov, V. P. Formation of a protein corona around nanoparticles. Curr. Opin. Colloid Interface Sci. 41, 95-103 (2019).

118. Ke, P. C., Lin, S., Parak, W. J., Davis, T. P. \& Caruso, F. A decade of the protein corona. ACS Nano 11, 11773-11776 (2017).

119. Cai, R. et al. Corona of thorns: the surface chemistry-,ediated protein corona perturbs the recognition and immune response of macrophages. ACS Appl. Mater. Interfaces 12, 1997-2008 (2020).

120. Thapa, S. \& Prasanna, R. Prospecting the characteristics and significance of the phyllosphere microbiome. Ann. Microbiol. 68, 229-245 (2018).

121. Mohanram, S. \& Kumar, P. Rhizosphere microbiome: revisiting the synergy of plant-microbe interactions. Ann. Microbiol. 69, 307-320 (2019).

122. Bai, Y. et al. Functional overlap of the Arabidopsis leaf and root microbiota. Nature 528, 364-369 (2015).

123. Hassani, M. A., Durán, P. \& Hacquard, S. Microbial interactions within the plant holobiont. Microbiome 6, 58 (2018).

124. Busby, P. E. et al. Research priorities for harnessing plant microbiomes in sustainable agriculture. PLoS Biol. 15, e2001793 (2017).

125. Müller, D. B., Vogel, C., Bai, Y. \& Vorholt, J. A. The plant microbiota: systems-level insights and perspectives. Annu. Rev. Genet. 50, 211-234 (2016).

126. Kozawa, D. et al. A fiber optic interface coupled to nanosensors: applications to protein aggregation and organic molecule guantification. ACS Nano 14, 10141-10152 (2020).

127. Di, W. \& Clark, H. A. Optical nanosensors for: in vivo physiological chloride detection for monitoring cystic fibrosis treatment. Anal. Methods 12, 1441-1448 (2020).

128. Parikh, S. J., Goyne, K. W., Margenot, A. J., Mukome, F. N. D. \& Calderón, F. J. in Advances in Agronomy Vol. 126 (Academic Press Inc., 2014).

129. Wasson, A., Bischof, L., Zwart, A. \& Watt, M. A portable fluorescence spectroscopy imaging system for automated root phenotyping in soil cores in the field. J. Exp. Bot. 67, 1033-1043 (2016).

130. Tracy, S. R. et al. Crop Improvement from phenotyping roots: highlights reveal expanding opportunities. Trends Plant Sci. 25, 105-118 (2020).

131. $\mathrm{Gu}, \mathrm{K}$. et al. Crosskingdom growth benefits of fungus-derived phytohormones in Choy Sum. Preprint at bioRxiv https://doi. org/10.1101/2020.02.04.933770 (2020).

132. Polisetti, S., Bible, A. N., Morrell-Falvey, J. L. \& Bohn, P. W. Raman chemical imaging of the rhizosphere bacterium Pantoea sp. YR343 and its co-culture with Arabidopsis thaliana. Analyst 141, 2175-2182 (2016).

133. Singer, E., Wagner, M. \& Woyke, T. Capturing the genetic makeup of the active microbiome in situ. ISME J. 11, 1949-1963 (2017).

134. Gehan, M. A. et al. PlantCV v2: image analysis software for high-throughput plant phenotyping. PeerJ 5, e4088 (2017).

135. Rodriguez, P. A. et al. Systems biology of plant-microbiome interactions. Mol. Plant 12, 804-821 (2019).

136. Toju, H. et al. Core microbiomes for sustainable agroecosystems. Nat. Plants 4, 247-257 (2018).

137. Ballaré, C. L. \& Pierik, R. The shade-avoidance syndrome: multiple signals and ecological consequences. Plant. Cell Environ. 40, 2530-2543 (2017).

138. Tang, Y.-J. \& Liesche, J. The molecular mechanism of shade avoidance in crops - how data from Arabidopsis can help to identify targets for increasing yield and biomass production. J. Integr. Agr. 16, 1244-1255 (2017)

139. Wille, W., Pipper, C. B., Rosenqvist, E., Andersen, S. B. \& Weiner, J. Reducing shade avoidance responses in a cereal crop. AoB Plants 9 plx039 (2017).

140. Casal, J. J. Shade avoidance. Arab. B. 10, e0157 (2012).

141. Devlin, P. F., Yanovsky, M. J. \& Kay, S. A. A genomic analysis of the shade avoidance response in Arabidopsis. Plant Physiol. 133, 1617-1629 (2003).
142. Rolauffs, S., Fackendahl, P., Sahm, J., Fiene, G. \& Hoecker, U. Arabidopsis COP1 and SPA genes are essential for plant elongation but not for acceleration of flowering time in response to a low red light to far-red light ratio. Plant Physiol. 160, 2015-2027 (2012).

143. Asseng, S. et al. Rising temperatures reduce global wheat production. Nat. Clim. Change 5, 143-147 (2015).

144. Pereira, A. Plant abiotic stress challenges from the changing environment. Front. Plant Sci. 7, 1123 (2016).

145. Elad, Y. \& Pertot, I. Climate change impacts on plant pathogens and plant diseases. J. Crop Improv. 28, 99-139 (2014)

146. Piao, S. et al. The impacts of climate change on water resources and agriculture in China. Nature 467, 43-51 (2010).

147. Biermacher, J. T., Brorsen, B. W., Epplin, F. M., Solie, J. B. \& Raun, W. R. The economic potential of precision nitrogen application with wheat based on plant sensing. Agric. Econ. 40, 397-407 (2009).

148. Polo, E. \& Kruss, S. Impact of redox-active molecules on the fluorescence of polymer-wrapped carbon nanotubes. J. Phys. Chem. C 120, 3061-3070 (2016).

149. Wang, L., Luo, W., Sun, X. \& Qian, C. Changes in flavor-relevant compounds during vine ripening of tomato fruit and their relationship with ethylene production. Hortic. Environ. Biotechnol. 59, 787-804 (2018).

150. Klee, H. J. \& Giovannoni, J. J. Genetics and control of tomato fruit ripening and quality attributes. Annu. Rev. Genet. 45, 41-59 (2011).

151. Akhatou, I. \& Fernández-Recamales, Á. Nutritional and nutraceutical quality of strawberries in relation to harvest time and crop conditions. J. Agric. Food Chem. 62, 5749-5760 (2014).

152. Lowry, G. V., Avellan, A. \& Gilbertson, L. M. Opportunities and challenges for nanotechnology in the agri-tech revolution. Nat. Nanotechnol. 14, 517-522 (2019).

153. Kah, M., Kookana, R. S., Gogos, A. \& Bucheli, T. D. A critical evaluation of nanopesticides and nanofertilizers against their conventional analogues. Nat. Nanotechnol. 13, 677-684 (2018).

154. Falinski, M. M. et al. A framework for sustainable nanomaterial selection and design based on performance, hazard, and economic considerations. Nat. Nanotechnol. 13, 708-714 (2018).

155. Hofmann, T. et al. Technology readiness and overcoming barriers to sustainably implement nanotechnology-enabled plant agriculture. Nat. Food 1, 416-425 (2020).

156. Eschen, R. et al. Safeguarding global plant health: the rise of sentinels. J. Pest Sci. 92, 29-36 (2019).

157. Kenis, M. et al. Sentinel nurseries to assess the phytosanitary risks from insect pests on importations of live plants. Sci. Rep. 8, 11217 (2018).

158. Radoglou-Grammatikis, P., Sarigiannidis, P., Lagkas, T. \& Moscholios, I. A compilation of UAV applications for precision agriculture. Comput. Networks 172, 107148 (2020).

159. Santesteban, L. G. et al. High-resolution UAV-based thermal imaging to estimate the instantaneous and seasonal variability of plant water status within a vineyard. Agric. Water Manag. 183, 49-59 (2017).

160. Saiz-Rubio, V. \& Rovira-Más, F. From smart farming towards agriculture 5.0: a review on crop data management. Agronomy 10, 207 (2020).

\section{Acknowledgements}

The authors are grateful for the funding and support from the MIT-Singapore SMART programme, specifically the Disruptive and Sustainable Technology for Agricultural Precision (DiSTAP) integrated research group, and the National Research Foundation, Singapore (NRF-CRP16-2015-04 to N.I.N). T.T.S.L. was supported on a graduate fellowship by the Agency of Science, Research and Technology, Singapore. We thank B Skrip (Massachusetts Institute of Technology) for creating Fig. 2 in this manuscript.

\section{Author contributions}

T.T.S.L., N.-H.C. and M.S.S. led the writing of this manuscript. T.T.S.L., R.S., I.-C.J., B.S.P., N.I.N., M.H.W., G.P.S., R.J.R., O.S., K.S., N.-H.C. and M.S.S. contributed to the writing of specific sections, critical reading of the manuscript and the reviewing of appropriate references.

\section{Competing interests}

The authors declare no competing interests.

\section{Additional information}

Correspondence should be addressed to N.-H.C. or M.S.S.

Reprints and permissions information is available at www.nature.com/reprints.

Publisher's note Springer Nature remains neutral with regard to jurisdictional claims in published maps and institutional affiliations.

(C) Springer Nature Limited 2020 